\title{
Bacteriostatic and Virostatic Activities of Mushrooms: A Mini-review
}

\author{
Li YC, Li Y, Guo HY, Wang CY* and Yang XW*
}

School of Life Sciences, Jilin University, Changchun, 130012, China

Li YC, Li Y, Guo HY, Wang CY and Yang XW 2021 - Bacteriostatic and Virostatic Activities of Mushrooms: A Mini-review. Fungal Biotec 1(1), 1-12, Doi 10.5943/FunBiotec/1/1/1

\begin{abstract}
In this study, we discuss the various bacteriostatic and antiviral effects of mushrooms and their potential mechanisms of action. During this research, we included both Chinese and international studies. Mushrooms and their active constituents, such as fatty acids, polysaccharides and proteins, were found to exert significant antibacterial and antiviral effects. These antimicrobial mechanisms appear to be similar to those of penicillin, which prevents bacterial growth by inhibiting bacterial cell wall synthesis. In contrast, the antiviral effects likely result from nonspecific enhancement of immune function and regulation and increase of cellular repair. However, at present, research on the effective ingredients and relevant mechanisms of action of mushrooms is lacking. Thus, further research is warranted.
\end{abstract}

Key words - active ingredients - antibacterial - antiviral - fungus - mechanism

\section{Introduction}

Fungi are nucleated, sporogenic and chloroplast-free eukaryotes. Mushrooms are a specific type of fungi, some of which are known to be both delicious and nutrient-rich (Reis et al. 2012). The class Basidiomycetes encompasses many mushrooms that are considered delicacies. These mushrooms have high nutritional and functional value, and many are also recognized as nutraceutical foods. These mushrooms are of considerable value due to their organoleptic merit, potential medicinal properties and economic significance (Elena et al. 2015). Moreover, mushrooms contain a wide variety of amino acids, minerals, proteins and vitamins, and can thus benefit our health (Wang et al. 2014). They have been described as an "appetizing" food source that "are beneficial to the stomach, dissolve phlegm and help regulate the body" (Friedman 2016). In traditional Chinese medicine, it is believed that mushrooms can promote gut health, dissipate phlegm and treat vomiting and diarrhea (De Silva et al. 2012, Reis et al. 2017, Wasser 2017). Modern medical studies have reported that mushrooms can effectively inhibit the growth of Escherichia coli, Salmonella typhi and Staphylococcus aureus and exert bacteriostatic and antiinflammatory effects (Muszynska et al. 2018). For example, mushrooms have been found to exert a curative effect against Vesicular stomatitis virus Latin. Thus, mushrooms are anti-bacterial and anti-viral and thus have high medical value (Wasser 2010). In this review, we will summarize the various bacteriostatic and virostatic activities of fungi and discuss their potential mechanisms of action.

\section{Bacteriostatic and antiviral effects of mushrooms}

Mushrooms can enhance digestion and support liver function (Martel et al. 2017). In recent

Submitted 1 March 2021, Accepted 3 June 2021, Published 30 June 2021

Corresponding Author: Xuewei Yang - e-mail: yangxuewei@jlu.edu.cn, Chunyue Wang - e-mail:

chunyue19@mails.jlu.edu.cn 
years, studies have reported that mushrooms possess lipotropic, antithrombotic, antibacterial and antiviral activities.

As an edible and medicinal fungus, Agaricus blazei Murill (AbM) is recognized as being both savory and nutritious, containing various nutritional and biologically active substances. Aqueous $\mathrm{AbM}$ extract has been reported to have antibacterial and antifungal properties, particularly against $E$. coli and $S$. aureus. It has also been found to display a strong inhibitory effect on molds. Moreover, aqueous or alcohol AbM extracts and AbM polysaccharides have been found to exert inhibitory effect on herpesviruses and poliovirus (Ye \& Lin 2001, Wang et al. 2008). Lentinus edodes, commonly known as the shiitake mushroom, is a fungus of the basidiomycetes family that has a mild and sweet taste and is believed to aid digestion. The medicinal properties and functions of $L$. edodes have been reported by pharmacists. Lentinan, a polysaccharide produced by L. edodes, is a biologically active substance that possesses antibacterial, antitumor and antiviral properties. It can effectively inhibit the growth of microorganisms including adenovirus, Bacillus subtilis, E. coli, herpes simplex virus, influenza virus, respiratory syncytial virus, Salmonella typhimurium, $S$. aureus and S. haemolyticus. Additionally, it has been reported to delay the onset of pathological cellular changes and has no toxic side effects (Zhang et al. 2006, 2007, Zou et al. 2007, Hou \& Zhang 2015, Tang et al. 2018). Amanita verna (AV) inhibits the pathogens responsible for poplar canker disease, such as Cytospora chrysosperma (Ji et al. 2012). Russula is a large mycorrhizal fungus that is nutritious and has been reported to have beneficial effects on the circulatory system. According to recent findings, some fungi display strong antibacterial activity against $B$. subtilis, $E$. coli and Pseudomonas aeruginosa (Chen et al. 2008). Pleurotus eryngii is a rare edible fungus that has both dietotherapuetic and medicinal properties. Both the liquid fermentation broth and bran extract of $P$. eryngii have been reported to inhibit the growth of three common pathogenic bacteria: $E$. coli, $S$. enteritidis and $S$. aureus, among which $S$. aureus experiences the most significant inhibitory effects (Dong et al. 2019). Inonotus hispidus (Bull.) P. Karst. has been used in traditional medicine to treat cancer, diabetes and dyspepsia. Numerous studies have confirmed the antimicrobial, antioxidative, antiproliferative, antiviral, anti-cytotoxic biological and immunomodulatory activities of extracts derived from this species. Moreover, it has been found to be effective against gram-positive bacteria (Bacillus cereus), gram-negative bacteria ( $P$. aeruginosa) and fungi (Candida albicans) when analyzed using the agar diffusion method (Angelini et al. 2019). Hypsizygus tessulatus, commonly known as the Shimeji mushroom, contains glucans, niacin and vitamins B and D. Previously, the mushroom was named Hypsizygus marmoreus and was reported to contain a wide range of biologically active compounds that potentially have a variety of medicinal applications. Currently, studies are examining the antibacterial and antifungal activities of its extract (Chowdhury et al. 2015). Triterpenoids obtained from Ganoderma spp. such as applanoxidic acid G, ganodermadiol and lucidadiol, have also displayed antiviral activity in vitro against infuenza virus type A, while ganodermadiol also inhibited the proliferation of herpes simplex virus (HSV) type 1. Eleven fungal species including Daedaleopsis confragosa, Datronia mollis, Laricifomes officinalis, Ischnoderma benzoinum, Trametes betulina, and Trametes gibbosa, have been reported to produce effective antiviral compounds. Agrocybe salicacola produces agrocybone, a sesquiterpene with weak antiviral activity against respiratory syncytial virus (Dasgupta \& Acharya 2019, Suwannarach et al. 2020).

Additionally, several studies have shown that AbM, Agrocybe aegerita, Coprinus comatus and fungi of the Lentinus and Pleurotus genera produced strong bacteriostatic and antiviral effects against B. subtilis, coxsackie group B virus, E. coli, and S. aureus (Liu et al. 2003, Gui 2004, Dai et al. 2007, Lin et al. 2014) (Table 1). These findings further support the hypothesis that mushrooms can exert both bacteriostatic and antiviral effects.

\section{Active ingredients in mushrooms}

Different species of mushroom contain different active ingredients. The strength of an active ingredient is proportional to its concentration. The same active ingredient may exert several different effects, some of which may depend on the combined activity of multiple substances. 
Polysaccharides produced by AbM have an inhibitory effect on poliovirus (PV-1) (Wang et al. 2008). Compounds derived from the esterification of sesquiterpene alcohols produced by Lactarius trivialis can reduce HSV titers of herpes simplex virus (Gui 2004). Lentinan inhibits the coxsackie virus, HSV and influenza virus, with these inhibitory effects being proportional to the lentinan concentration (Zhang et al. 2006, 2007, Zou et al. 2007). Additionally, sulfated lentinan has a significantly enhanced inhibitory effect on the infection of cells by infectious bursal disease virus, infectious bronchitis virus and Newcastle disease virus (Tang et al. 2018).

Polysaccharides produced by Agaricus crocopeplus Berk. \& Broome, L. edodes and Pholiota nameko (T. Ito) S. Ito \& S. Imai are effective against E. coli and S. aureus (Liu 2009). Furthermore, the acidic polysaccharides found in Agaricus bisporus are effective against Fusobacterium nucleatum, Porphyromonas gingivalis and Prevotella intermedia (Wei et al. 2007). Lastly, an acidic polysaccharide fraction obtained from the edible mushroom Pleurotus eous (Berk.) Sacc. was found to exert an inhibitory effect on B. subtilis, E. coli, Klebsiella pneumoniae and S. aureus (Gunasekaran et al. 2021). L-amino acid oxidases (LAOs) obtained from the fruiting bodies of Amanita phalloides (ApLAO) and Infundibulicybe geotropa ( $C g \mathrm{LAO})$ possess antibacterial properties against Agrobacterium tumefaciens, Dickeya chrysanthemi, Enterobacter spp., E. coli, Lactococcus lactis, Pectobacterium atrosepticum, Pectobacterium carotovorum, Ralstonia mannitolilytica, Ralstonia solanacearum and Xanthomonas arboricola (Jerica et al. 2020). Additionally, a novel antibacterial protein with a molecular mass of $44 \mathrm{kDa}$ was isolated from dried fruiting bodies of the wild mushroom Clitocyb sinopica (Fr.) Gill. It was determined that this protein possesses potent antibacterial activity against Agr. rhizogenes, Agr. tumefaciens, Agr. vitis, $X$. oryzae and X. malvacearum (Zhang et al. 2016). In one study, researchers induced the expression of members of two putative antibacterial peptide and protein families in Coprinopsis cinerea, cysteine-stabilized xp-defensins (Csxp-defensins) and GH24-type lysozymes, and purified the proteins; their analysis confirmed the antibacterial properties of these proteins against B. subtilis and E. coli (Kombrink et al. 2019). Psathyrelloic acid, a novel monocyclic diterpenoid isolated from Psathyrella candolleana cultures, has displayed effectiveness against $S$. aureus (Liu et al. 2019). Benzoic (4-OH-benzoic, protocatechuic, syringic and vanillic) and cinnamic (caffeic, ferulic and $p$-coumaric) acid derivatives obtained from 31 Polish's mushrooms displayed obvious antibacterial effects against $B$. subtilis, E. coli, K. pneumoniae, Micrococcus luteus, P. aeruginosa, Proteus mirabilis, S. aureus and S. epidermidis (Nowacka et al. 2017).

Fatty acids extracted from $A$. bisporus have displayed a significant inhibitory effect against gram-positive bacteria, while proteins from this species can inhibit methicillin resistant $S$. aureus and $S$. aureus (Tehrani et al. 2012, Zheng et al. 2016). Furthermore, polysaccharides produced by A. aegerita have an inhibitory effect against B. subtilis, E. coli and $S$. aureus (Xin et al. 2011). Amanita virgineoides bas and Am. rufoferruginea hongo alcohol extracts have been reported to exert an inhibitory effect on mycelial growth of Fusarium graminearum, Pestalotia theae and Septogloeum mori (Tang et al. 2015). Fermentation filtrate extracts from Am. muscaria, Am. squama and Lactarius velutipes have a significant inhibitory effect on the mycelial growth and spore germination of Cytospora chrysosperm and Sphaeropsis sapinea, but this effect varies with different extraction methods (Song \& Ji 2005, Qi \& Song 2006). High-performance liquid chromatography and electron spray ionization-mass spectrum methods are commonly used to separate and identify $\alpha$-amamanotoxin ( $\alpha$-AMA), $\beta$-manotoxin ( $\beta$-AMA) and dihydroxy phalloidin (PHD) from Am. pallidorosea P. Zhang \& Zhu L. Yang, which are known to have an inhibitory effect on C. albicans (Wang et al. 2011). Hericium erinaceus polysaccharide can inhibit the growth of E. coli (Chen et al. 2012). Similarly, Tricholoma matsutake polysaccharide exerts a significant concentration-dependent antibacterial effect on S. aureus (Hu \& Liu 2006). Gallic acid produced by Cyclosporium has a strong inhibitory effect on B. cereus, B. subtilis, C. albicans, E. coli, Salmonella paratyphi and S. aureus, and a weaker inhibitory effect on M. luteus (Wang et al. 2019). Furthermore, L. edodes coumarin, disulfide derivatives, methionine and thionine can inhibit $C$. albicans, $P$. intermedia and S. mutans (Hirasawa et al. 1999, Hatvani 2001). 
Table 1 Summary of bacteriostatic and virostatic activities of mushrooms

\begin{tabular}{|c|c|c|c|}
\hline Mushroom & Pathogenic bacterium & Effective constituent & References \\
\hline Amanita fritillaria & $\begin{array}{l}\text { Fusarium graminearum }\left(24.86 \% \%^{\mathrm{a}}\right) \text {, Pestalotiopsis theae } \\
(7.62 \% \mathrm{a}) \text {, Septogleum mori }\left(24.58 \% \%^{\mathrm{a}}\right)\end{array}$ & Ethanol extract & Tang et al. (2015) \\
\hline $\begin{array}{l}\text { Amanita } \\
\text { hemibapha }\end{array}$ & $\begin{array}{l}\text { Fusarium graminearum }(22.7 \% \text { a }) \text {, Pestalotiopsis theae } \\
\left(7.62 \%^{\mathrm{a}}\right) \text {, Septogleum mori }\left(22.5 \%^{\mathrm{a}}\right)\end{array}$ & Ethanol extract & Tang et al. (2015) \\
\hline $\begin{array}{l}\text { Amanita } \\
\text { manginiana }\end{array}$ & $\begin{array}{l}\text { Fusarium graminearum }\left(22.16 \% \%^{\mathrm{a}}\right) \text {, Pestalotiopsis theae } \\
\left(6.73 \%^{\mathrm{a}}\right) \text {, Septogleum mori }\left(20.83 \%^{\mathrm{a}}\right)\end{array}$ & Ethanol extract & Tang et al. (2015) \\
\hline $\begin{array}{l}\text { Amanita } \\
\text { rufoferruginea }\end{array}$ & $\begin{array}{l}\text { Fusarium graminearum }(65.95 \% \text { a }) \text {, Pestalotiopsis theae } \\
(77.58 \% \text {, Septogleum mori }(85.00 \% \text { a })\end{array}$ & Ethanol extract & Tang et al. (2015) \\
\hline Agaricus bisporus & $\begin{array}{l}\text { Bacillus cereus }\left(59.52^{\mathrm{c}}, 10.75^{\mathrm{b}}\right), \text { Enterococcus faecalis } \\
\left(>20^{\mathrm{c}}\right), \text { Escherichia coli }\left(59.52^{\mathrm{c}}\right) \text {, methicillin resistant } \\
\text { Staphylococcus aureus }\left(10^{\mathrm{c}}\right) \text {, methicillin sensitive } \\
\text { Staphylococcus aureus }\left(10^{\mathrm{c}}\right), \text { Pseudomonas aeruginosa } \\
\left(59.52^{\mathrm{c}},>20^{\mathrm{c}}\right), \text { Salmonella typhimurium }\left(59.52^{\mathrm{c}}, 10.75^{\mathrm{b}}\right) \text {, } \\
\text { Staphylococcus aureus }\left(59.52^{\mathrm{c}},>20^{\mathrm{c}}\right)\end{array}$ & $\begin{array}{l}\text { Ethanol extract, } \\
\text { methanol extracts }\end{array}$ & $\begin{array}{l}\text { Taofiq et al. } \\
\text { (2016), Fogarasi et } \\
\text { al. (2020), Melinda } \\
\text { et al. (2020) }\end{array}$ \\
\hline Agaricus blazei & 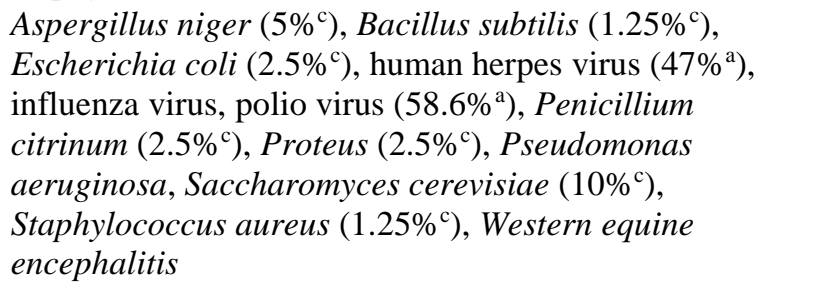 & $\begin{array}{l}\text { Water extract, ethanol } \\
\text { extract, polysaccharide }\end{array}$ & $\begin{array}{l}\text { Ye \& Lin }(2001) \\
\text { Hetland et al. } \\
(2021)\end{array}$ \\
\hline $\begin{array}{l}\text { Agaricus } \\
\text { crocopeplus }\end{array}$ & $\begin{array}{l}\text { Escherichia coli }\left(2.5^{\mathrm{c}}\right), \text { Salmonella }\left(2.5^{\mathrm{c}}\right) \text {, } \\
\text { Staphylococcus aureus }\left(5^{\mathrm{c}}\right)\end{array}$ & Water extract & Liu (2009) \\
\hline Agaricus gennadii & $\begin{array}{l}\text { Bacillus cereus }\left(8.01^{\mathrm{b}}\right) \text {, Bacillus subtilis }\left(7.93^{\mathrm{b}}\right) \text {, } \\
\text { Candida albicans }\left(7.62^{\mathrm{b}}\right) \text {, Escherichia coli }\left(7.05^{\mathrm{b}}\right) \text {, } \\
\text { Micrococcus luteus }\left(6.38^{\mathrm{b}}\right) \text {, Salmonella paratyphi B } \\
\left(9.21^{\mathrm{b}}\right), \text { Staphylococcus aureus }\left(9.02^{\mathrm{b}}\right)\end{array}$ & Gallic acid & Wang et al. (2019) \\
\hline Agaricus sp. & Pseudomonas aeruginosa, Staphylococcus aureus & Water extract & $\begin{array}{l}\text { Mariselvi \& } \\
\text { Ninganna (2018) }\end{array}$ \\
\hline $\begin{array}{l}\text { Agaricus } \\
\text { xanthodermus }\end{array}$ & Pseudomonas aeruginosa, Staphylococcus aureus & Water extract & $\begin{array}{l}\text { Mariselvi \& } \\
\text { Ninganna (2018) }\end{array}$ \\
\hline Amanita muscaria & Cytospora chrysosperm $\left(92.42 \%{ }^{a}\right)$ & $\begin{array}{l}\text { Fermentation filtrate } \\
\text { extract }\end{array}$ & $\begin{array}{l}\text { Song \& Ji (2005), } \\
\text { Qi \& Song (2006) }\end{array}$ \\
\hline $\begin{array}{l}\text { Amanita } \\
\text { pallidorosea }\end{array}$ & Candida albicans $\left(10.16 \% \%^{\mathrm{a}}, 6.89 \%^{\mathrm{a}}, 11.1 \%^{\mathrm{a}}, 8.37 \%^{\mathrm{a}}\right)$ & $\begin{array}{l}\text { Ethanol extract, } \alpha- \\
\text { AMA, } \beta \text {-AMA, PHD }\end{array}$ & Wang et al. (2011) \\
\hline $\begin{array}{l}\text { Amanita } \\
\text { phalloides }\end{array}$ & $\begin{array}{l}\text { Agrobacterium tumefaciens, Dickeya chrysanthemi, } \\
\text { Enterobacter sp., Escherichia coli, Lactococcus lactis, } \\
\text { Pectobacterium atrosepticum, Pectobacterium } \\
\text { carotovorum, Ralstonia mannitolilytica, Ralstonia } \\
\text { solanacearum, Xanthomonas arboricola }\end{array}$ & L-amino acid oxidases & Jerica et al. (2020) \\
\hline Amanita sp. & Cytosporium chrysosporium $(68.27 \%$ a $)$ & Ethanol extract & Ji et al. (2012) \\
\hline $\begin{array}{l}\text { Amanita } \\
\text { virgineoides Bas }\end{array}$ & $\begin{array}{l}\text { Fusarium graminearum schw. }(87.03 \% \text { a }) \text {, Pestalotiopsis } \\
\text { theae }\left(\text { Sawada) Steyaert }\left(83.86 \%^{\mathrm{a}}\right) \text {, Septogleum mori Bri }\right. \\
\text { et Cav }\left(72.50 \%^{\mathrm{a}}\right)\end{array}$ & Ethanol extract & Tang et al. (2015) \\
\hline Amanita virosa & $\begin{array}{l}\text { Cytospora chrysosperm }(92.42 \% \text { a }) \text {, Sphaeropsis sapinea } \\
\left(12.41 \%^{\mathrm{a}}\right)\end{array}$ & $\begin{array}{l}\text { Fermentation filtrate } \\
\text { extract }\end{array}$ & $\begin{array}{l}\text { Song \& Ji (2005), } \\
\text { Qi \& Song (2006) }\end{array}$ \\
\hline Boletinus paluster & $\begin{array}{l}\text { Agrobacterium tumefaciens }\left(15^{\mathrm{b}}\right) \text {, Xanthomonas oryzae } \\
\left(7.5^{\mathrm{b}}\right) \text {, Xanthomonas oryzae }\left(11^{\mathrm{b}}\right) \text {, Xanthomonas } \\
\text { campestris }\left(5.5^{\mathrm{b}}\right)\end{array}$ & Water extract & Zheng et al. (2010) \\
\hline Boletus edulis & $\begin{array}{l}\text { Bacillus cereus }\left(12.04^{\mathrm{b}}, 28.34^{\mathrm{c}}\right), \text { Escherichia coli } \\
\left(28.34^{\mathrm{c}}\right) \text {, Pseudomonas aeruginosa }\left(11.98^{\mathrm{b}}, 28.34^{\mathrm{c}}\right) \text {, } \\
\text { Salmonella typhimurium }\left(13.49^{\mathrm{c}}\right), \text { Staphylococcus aureus } \\
\left(28.34^{\mathrm{c}}\right)\end{array}$ & Methanolic extracts & $\begin{array}{l}\text { (Fogarasi et al. } \\
(2020), \text { Melinda et } \\
\text { al. (2020) }\end{array}$ \\
\hline $\begin{array}{l}\text { Cantharellus } \\
\text { cibarius }\end{array}$ & $\begin{array}{l}\text { Bacillus cereus }\left(59.52^{c}\right), \text { Escherichia coli }\left(59.52^{c}\right), \\
\text { Pseudomonas aeruginosa }\left(59.52^{c}\right), \text { Salmonella } \\
\text { typhimurium }\left(59.52^{c}\right), \text { Staphylococcus aureus }\left(59.52^{c}\right)\end{array}$ & Methanolic extracts & $\begin{array}{l}\text { Liu et al. (2003), } \\
\text { Melinda et al. } \\
\text { (2020) }\end{array}$ \\
\hline Clitocybe rivulosa & Sphaeropsis sapinea $(45.92 \%$ a $)$ & $\begin{array}{l}\text { Fermentation filtrate } \\
\text { extract }\end{array}$ & Qi \& Song (2006) \\
\hline
\end{tabular}


Table 1 Continued.

\begin{tabular}{|c|c|c|c|}
\hline Types of Fungus & Pathogenic Bacterium & Effective constituent & References \\
\hline Clitocybe sinopica & $\begin{array}{l}\text { Agrobacterium rhizogenes, Agrobacterium tumefaciens } \\
\left(16^{\mathrm{b}}\right), \text { Agrobacterium vitis, Xanthomonas oryzae }\left(8.5^{\mathrm{b}}\right) \text {, } \\
\text { Xanthomonas oryzae }\left(10.5^{\mathrm{b}}\right)\end{array}$ & Water extract, protein & Zheng et al. (2010) \\
\hline $\begin{array}{l}\text { Coprinellus } \\
\text { disseminatus }\end{array}$ & Staphylococcus aureus & water extract & $\begin{array}{l}\text { Mariselvi \& } \\
\text { Ninganna (2018) }\end{array}$ \\
\hline $\begin{array}{l}\text { Coprinopsis } \\
\text { cinerea }\end{array}$ & Bacillus subtilis, Escherichia coli & $\begin{array}{l}\text { Cysteine stabilized } \alpha \beta \text { - } \\
\text { defensins, GH24-type } \\
\text { lysozymes }\end{array}$ & (Kombrink et al. \\
\hline Coprinus comatus & $\begin{array}{l}\text { Bacillus licheniformis }\left(6^{\mathrm{b}}\right) \text {, Bacillus subtilis }\left(3.0^{\mathrm{b}}\right) \text {, } \\
\text { Escherichia coli }\left(3.5^{\mathrm{b}}\right) \text {, Staphylococcus aureus }\left(4.5^{\mathrm{b}}\right)\end{array}$ & Water extract & $\begin{array}{l}\text { Zou et al. (2012), } \\
\text { Sadi et al. (2015) }\end{array}$ \\
\hline $\begin{array}{l}\text { Phallus impudicus } \\
\text { (=Dictyophora } \\
\text { indusiata (Vent.ex } \\
\text { Pers) Fisch) }\end{array}$ & $\begin{array}{l}\text { Escherichia coli }\left(12.5^{c}\right), \text { Listeria monocytogenes }\left(25^{c}\right) \text {, } \\
\text { Salmonella enteritidis }\left(25^{c}\right) \text {, Staphylococcus aureus } \\
\left(25^{c}\right) \text {, Vibrio parahaemolyticus }\left(12.5^{c}\right)\end{array}$ & Alcohol extract & Zheng et al. (2013) \\
\hline $\begin{array}{l}\text { Flammulina } \\
\text { velutipes }\end{array}$ & $\begin{array}{l}\text { Aerobic bacillus }\left(36.3 \%{ }^{\mathrm{a}}\right) \text {, Bacillus subtilis }\left(20.4 \%^{\mathrm{a}}\right) \text {, } \\
\text { Escherichia coli }(28.6 \% \text {, Saccharomyces cerevisiae } \\
\left(37.5 \%^{\mathrm{a}}\right) \text {, Staphylococcus aureus }\left(57.2 \% \%^{\mathrm{a}}\right) \text {, Waxy } \\
\text { bacillus }\left(42.0 \%^{\mathrm{a}}\right)\end{array}$ & Ester extract & Li et al. (2007) \\
\hline $\begin{array}{l}\text { Fomitopsis } \\
\text { pinicola }\end{array}$ & $\begin{array}{l}\text { Aspergillus fumigates }\left(3.2^{\mathrm{c}}\right) \text {, Bacillus subtilis }\left(0.8^{\mathrm{c}}\right) \text {, } \\
\text { Candida albicans }\left(3.2^{\mathrm{c}}\right), \text { Escherichia coli }\left(1.6^{\mathrm{c}}\right) \text {, } \\
\text { Klebsiella pneumoniae }\left(12.8^{\mathrm{c}}\right) \text {, Penicillium chrysogenum } \\
\left(3.2^{\mathrm{c}}\right) \text {, Proteus vulgaris }\left(0.8^{\mathrm{c}}\right) \text {, Pseudomonas aeruginosa } \\
\left(1.6^{\mathrm{c}}\right), \text { Saccharomyces cerevisiae }\left(1.6^{\mathrm{c}}\right) \text {, Staphylococcus } \\
\text { aureus }\left(1.6^{\mathrm{c}}\right)\end{array}$ & Ethyl acetat & Pala et al. (2019) \\
\hline Ganoderma sp. & Pseudomonas aeruginosa, Staphylococcus aureus & Wate & $\begin{array}{l}\text { Mariselvi } \\
\text { Ninganna }\end{array}$ \\
\hline Grifola frondosa & $\begin{array}{l}\text { Enterovirus, Pseudomonas sp., Streptococcus } \\
\text { pneumoniae }\end{array}$ & ccharide, water & $\begin{array}{l}\text { Hetland et al. } \\
(2021)\end{array}$ \\
\hline $\begin{array}{l}\text { Hericium } \\
\text { erinaceus }\end{array}$ & $\begin{array}{l}\text { Dengue virus, Escherichia coli }\left(7.0^{\mathrm{c}}\right) \text {, Helicobacter } \\
\text { pylori, Muscovy duck reovirus, Streptococcus mutans, } \\
\text { Streptococcus pneumoniae }\end{array}$ & Polysaccharide & $\begin{array}{l}\text { Chen et al. (2012), } \\
\text { Hetland et al. } \\
\text { (2021) }\end{array}$ \\
\hline $\begin{array}{l}\text { Hymenagaricus } \\
\text { sp. }\end{array}$ & Pseudomonas aeruginosa, Staphylococcus aureus & xtract & $\begin{array}{l}\text { Mariselvi \& } \\
\text { Ninganna (2018) }\end{array}$ \\
\hline $\begin{array}{l}\text { Hypsizigus } \\
\text { tessulatus }\end{array}$ & $\begin{array}{l}\text { Bacillus subtilis }\left(8^{c}\right) \text {, Candida albicans }\left(6^{c}\right) \text {, Escherichia } \\
\text { coli }\left(7^{c}\right) \text {, Klebsiella pneumoniae }\left(6^{c}\right) \text {, Pseudomonas } \\
\text { aeruginosa }\left(9^{c}\right), \text { Saccharomyces cerevisiae }\left(5^{c}\right), \\
\text { Salmonella typhi }\left(7^{c}\right), \text { Staphylococcus aureus }\left(7^{c}\right)\end{array}$ & Methanolic extracts & $\begin{array}{l}\text { Chowdhury et al. } \\
\text { (2015) }\end{array}$ \\
\hline $\begin{array}{l}\text { Infundibulicybe } \\
\text { geotropa }\end{array}$ & $\begin{array}{l}\text { Agrobacterium tumefaciens, Dickeya chrysanthemi, } \\
\text { Enterobacter sp., Escherichia coli, Lactococcus lactis, } \\
\text { Pectobacterium atrosepticum, Pectobacterium } \\
\text { carotovorum, Ralstonia mannitolilytica, Ralstonia } \\
\text { solanacearum, Xanthomonas arboricola }\end{array}$ & cid oxidases & Jerica et al. (2020) \\
\hline $\begin{array}{l}\text { Inonotus } \\
\text { andersonii }\end{array}$ & $\begin{array}{l}\text { Propionibacterium acnes }\left(0.2^{\mathrm{c}}\right), \text { Staphylococcus aureus } \\
\left(0.2^{\mathrm{c}}\right)\end{array}$ & Ethanol extract & $\begin{array}{l}\text { Tamrakar et al. } \\
\text { (2017) }\end{array}$ \\
\hline $\begin{array}{l}\text { Inonotus } \\
\text { clemensiae }\end{array}$ & $\begin{array}{l}\text { Propionibacterium acnes }\left(0.1^{\mathrm{c}}\right), \text { Staphylococcus aureus } \\
\left(0.1^{\mathrm{c}}\right)\end{array}$ & Ethanol extract & $\begin{array}{l}\text { Tamrakar et al. } \\
\text { (2017) }\end{array}$ \\
\hline $\begin{array}{l}\text { Inonotus } \\
\text { cuticularis }\end{array}$ & Staphylococcus aureus $\left(0.2^{\mathrm{c}}\right)$ & Ethanol extract & $\begin{array}{l}\text { Tamrakar et al. } \\
(2017)\end{array}$ \\
\hline \multicolumn{2}{|c|}{ 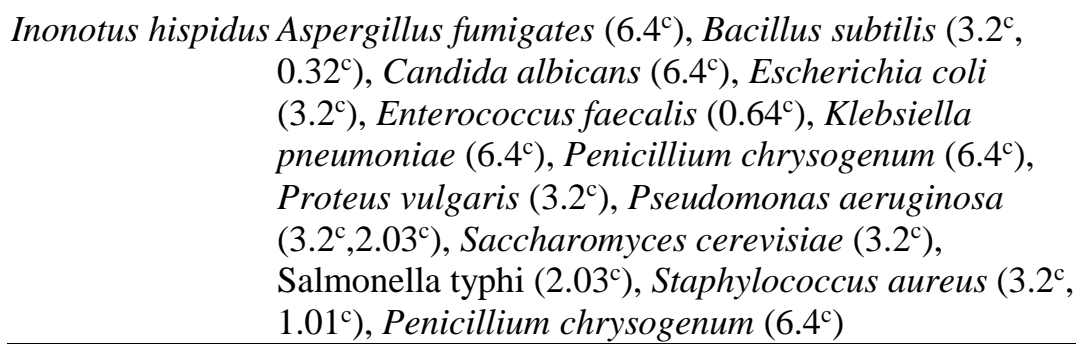 } & $\begin{array}{l}\text { Ethyl acetate extract, } \\
\text { methanol extracts }\end{array}$ & $\begin{array}{l}\text { Angelini et al. } \\
(2019), \text { Pala et al. } \\
(2019)\end{array}$ \\
\hline
\end{tabular}


Table 1 Continued.

\begin{tabular}{|c|c|c|c|}
\hline Types of Fungus & Pathogenic Bacterium & Effective constituent & References \\
\hline Inonotus obliguus & $\begin{array}{l}\text { Cytospora chrysosperm }\left(65^{c}\right), \text { Botryosphaeria laricina } \\
\left(60^{c}\right)\end{array}$ & $\begin{array}{l}\text { Ethanol extract, } \\
\text { polysaccharide }\end{array}$ & Zheng et al. (2010) \\
\hline $\begin{array}{l}\text { Lactarius } \\
\text { piperatus }\end{array}$ & $\begin{array}{l}\text { Bacillus cereus }\left(56.68^{c}\right), \text { Escherichia coli }\left(26.99^{c}\right) \text {, } \\
\text { Pseudomonas aeruginosa }\left(56.68^{c}\right), \text { Salmonella } \\
\text { typhimurium }\left(26.99^{c}\right), \text { Staphylococcus aureus }\left(56.68^{c}\right)\end{array}$ & Methanol extracts & $\begin{array}{l}\text { Melinda et al. } \\
(2020)\end{array}$ \\
\hline Lactarius uvidus & $\begin{array}{l}\text { Agrobacterium tumefaciens }\left(8.5^{\mathrm{b}}\right) \text {, Xanthomonas } \\
\text { campestris }\left(4.5^{\mathrm{b}}\right), \text { Xanthomonas oryzae }\left(5.5^{\mathrm{b}}\right)\end{array}$ & Water extract & Zhang et al. (2019) \\
\hline $\begin{array}{l}\text { Lactarius } \\
\text { vellereus }\end{array}$ & $\begin{array}{l}\text { Alternaria alternata }(92.71 \% \text { a }) \text {, Cytospora chrysosperm } \\
\left(92.42 \%^{\mathrm{a}}\right) \text {, Sphaeropsis sapinea }\left(58.89 \%^{\mathrm{a}}\right)\end{array}$ & $\begin{array}{l}\text { Fermentation filtrate } \\
\text { extract }\end{array}$ & $\begin{array}{l}\text { Song \& Ji (2005), } \\
\text { Qi \& Song (2006), } \\
\text { Ji et al. (2008) }\end{array}$ \\
\hline Lentinus edodes & $\begin{array}{l}\text { Adeno virus }(91 \% \text { a }) \text {, Agrobacterium tumefaciens }\left(7^{\mathrm{b}}\right) \text {, } \\
\text { Bacillus subtilis }\left(2.5^{\mathrm{b}}, 40^{\mathrm{c}}\right), \text { Bacillus typhi }\left(2.5^{\mathrm{c}}\right), \\
\text { Candida albicans }\left(40^{\mathrm{c}}\right) \text {, coxsackie virus }\left(91.1 \%^{\mathrm{a}}\right) \text {, echo } \\
\text { virus }\left(73 \% \%^{\mathrm{a}}\right), \text { Enterococcus faecalis }\left(5^{\mathrm{c}}\right), \text { Escherichia coli } \\
\left(0.625^{\mathrm{c}},>20^{\mathrm{c}}\right), \text { Hemolytic streptococcus }\left(1.25^{\mathrm{c}}\right) \text {, herpes } \\
\text { simplex virus }\left(89.4 \%^{\mathrm{a}}\right) \text {, influenza virus }\left(95.6 \%^{\mathrm{a}}\right), \\
\text { methicillin resistant Staphylococcus aureus }\left(2.5^{\mathrm{c}}\right) \text {, } \\
\text { methicillin sensitive Staphylococcus aureus }\left(2.5^{\mathrm{c}}\right) \text {, } \\
\text { Pasteurella multocida }\left(10^{\mathrm{c}}\right), \text { Pseudomonas aeruginosa }(>\end{array}$ & $\begin{array}{l}\text { Ethanol extract, } \\
\text { polysaccharide, water } \\
\text { extract }\end{array}$ & $\begin{array}{l}\text { Zhang et al. (2006, } \\
\text { 2007), Zou et al. } \\
\text { (2007), Liu (2009), } \\
\text { Zheng et al. } \\
\text { (2010), Wang \& } \\
\text { Wang (2011), Hou } \\
\& \text { Zhang (2015), } \\
\text { Taofiq et al. (2016) }\end{array}$ \\
\hline
\end{tabular}

$\left.20^{\mathrm{c}}\right)$, respiratory syncytial virus $(82 \%$ a), rota virus

$\left(85 \%^{\mathrm{a}}\right)$, Saccharomyces cerevisiae $\left(80^{\mathrm{c}}\right)$, Salmonella

paratyphi A $\left(5^{\mathrm{c}}\right)$, Salmonella typhimurium $\left(10^{\mathrm{c}}\right)$, Shigella

dysenteriae $\left(2.5^{\mathrm{c}}\right)$, Staphylococcus aureus $\left(2.5^{\mathrm{c}}\right)$,

Trichophyton rubrum $\left(160^{\mathrm{c}}\right)$, Xanthomonas campestris

$\left(6^{\mathrm{b}}\right)$, Xanthomonas oryzae $\left(5^{\mathrm{b}}\right)$

Lentinus tigrinus Agrobacterium tumefaciens $\left(8^{\mathrm{b}}\right)$, Aspergillus fumigates,

Bacillus licheniformis $\left(8^{\mathrm{b}}\right)$, Bacillus subtilis $\left(6^{\mathrm{b}}, 3.2^{\mathrm{c}}\right)$,

Candida albicans, Escherichia coli $\left(6^{\mathrm{b}}, 6.4^{\mathrm{c}}\right)$, Klebsiella

Ethyl acetate extract, Sadi et al. (2015),

pneumoniae $\left(12.8^{\mathrm{c}}\right)$, Penicillium chrysogenum, Proteus

vulgaris $(6.4 \mathrm{c})$, Pseudomonas aeruginosa $\left(6.4^{\mathrm{c}}\right)$,

Saccharomyces cerevisiae, Staphylococcus aureus $\left(6^{\mathrm{b}}\right.$,

$\left.6.4^{\mathrm{c}}\right)$

Lepiota Cytospora chrysosperm $\left(92.42 \%^{\mathrm{a}}\right)$, Sphaeropsis sapinea

cbypolaria $\quad\left(37.96 \%{ }^{\mathrm{a}}\right)$

Lepiota cristata Sphaeropsis sapinea $(38.15 \%$ )

$\mathrm{N}$-Hexane extract

Pala et al. (2019)

Lepista sordida Pseudomonas aeruginosa, Staphylococcus aureus

Fermentation filtrate

extract

Song \& Ji (2005),

Qi \& Song (2006)

extract

Water extract

Mariselvi \&

Ninganna (2018)

Leucopaxillus Agrobacterium tumefaciens $\left(12^{\mathrm{b}}\right)$, Bacillus subtilis (2 $\left.2^{\mathrm{b}}\right), \quad$ Water extract

giganteus Xanthomonas campestris $\left(6.5 \mathrm{~mm}^{\mathrm{b}}\right)$, Xanthomonas

oryzae $\left(9^{\mathrm{b}}\right)$, Xanthomonas oryzae $\left(14^{\mathrm{b}}\right)$

Lyophyllum sp. Pseudomonas aeruginosa, Staphylococcus aureus

Water extract

Mariselvi \& Ninganna (2018)

Lysurus mokusin Botrytis cinerea $(88.76 \%$ a)

Melanoleuca Agrobacterium tumefaciens $\left(14^{\mathrm{b}}\right)$, Xanthomonas

Ethanol extract

Zhang et al. (2019)

cognata

campestris $\left(6.5^{\mathrm{b}}\right)$, Xanthomonas oryzae $\left(10^{\mathrm{b}}\right)$,

Water extract

Zheng et al. (2010)

Xanthomonas oryzae pv. oryzicola (Fang et al.) Swing et

al. $\left(15^{\mathrm{b}}\right)$

Microporus spp. Candida albicans $\left(1.33^{\mathrm{c}}\right)$, Candida parapsilosis $\left(1.33^{\mathrm{c}}\right)$, Hot water extracts

(Kakamega forest) Escherichia coli $\left(1.67^{\mathrm{c}}\right)$, Klebsiella pneumoniae $\left(1.00^{\mathrm{c}}\right)$,

methicillin resistant Staphylococcus aureus $\left(1.00^{\mathrm{c}}\right)$,

Pseudomonas aeruginosa $\left(1.33^{\mathrm{c}}\right)$, Staphylococcus aureus

$\left(0.67^{\mathrm{c}}\right)$

Omphalotus

Pseudomonas aeruginosa, Staphylococcus aureus

Water extract

Mariselvi \&

olivascens

Pseudomonas aeruginosa

Water extract

Gebreselema et al. (2019) 
Table 1 Continued.

\begin{tabular}{|c|c|c|c|}
\hline Types of Fungus & Pathogenic Bacterium & Effective constituent & References \\
\hline Pholiota nameko & $\begin{array}{l}\text { Escherichia coli }\left(2.5^{\mathrm{c}}\right), \text { Salmonella }\left(2.5^{\mathrm{c}}\right) \text {, } \\
\text { Staphylococcus aureus }\left(5^{\mathrm{c}}\right)\end{array}$ & Water extract & Liu (2009) \\
\hline Pleurotus eryngii & $\begin{array}{l}\text { Escherichia coli }\left(14.38^{\mathrm{b}}\right), \text { Fusarium graminearum } \\
(87.45 \% \text { a }), \text { Fusarium oxysporum }(92.78 \% \text { a }) \text {, Salmonella } \\
\text { enteritidis }\left(9.31^{\mathrm{b}}\right) \text {, Staphylococcus aureus }\left(21.74^{\mathrm{b}}\right)\end{array}$ & $\begin{array}{l}\text { Fermentation broth, } \\
\text { polysaccharides }\end{array}$ & $\begin{array}{l}\text { Hou et al. (2016), } \\
\text { Dong et al. (2019) }\end{array}$ \\
\hline Pleurotus florida & $\begin{array}{l}\text { Bacillus subtilis }\left(13.8^{\mathrm{b}}\right), \text { Escherichia coli }\left(17.9^{\mathrm{b}}\right) \text {, } \\
\text { Pseudomonas aeruginosa }\left(16.2^{\mathrm{b}}\right), \text { Salmonella typhi } \\
\left(15.5^{\mathrm{b}}\right), \text { Streptococcus faecalis }\left(14.6^{\mathrm{b}}\right)\end{array}$ & Methanol extract & $\begin{array}{l}\text { Gashaw et al. } \\
(2020)\end{array}$ \\
\hline $\begin{array}{l}\text { Pleurotus } \\
\text { ostreatus }\end{array}$ & $\begin{array}{l}\text { Bacillus subtilis }\left(13.6^{\mathrm{b}}, 59.52^{\mathrm{c}}\right) \text {, Enterococcus faecalis } \\
\left(10^{\mathrm{c}}\right), \text { Escherichia coli }\left(19.5^{\mathrm{b}}, 59.52^{\mathrm{c}},>20^{\mathrm{c}}\right) \text {, methicillin } \\
\text { resistant Staphylococcus aureus }\left(2.5^{\mathrm{c}}\right), \text { methicillin } \\
\text { sensitive Staphylococcus aureus }\left(2.5^{\mathrm{c}}\right), \text { Mycobacterial } \\
\text { strains }\left(15^{\mathrm{c}}\right) \text {, Pseudomonas aeruginosa }\left(16.4^{\mathrm{b}}, 59.52^{\mathrm{c}}\right) \text {, } \\
\text { Salmonella typhi }\left(16.9^{\mathrm{b}}\right), \text { Streptococcus faecalis }\left(14.8^{\mathrm{b}} \text {, }\right. \\
\left.59.52^{\mathrm{c}},>20^{\mathrm{c}}\right)\end{array}$ & $\begin{array}{l}\text { Ethanol extract, } \\
\text { methanol extract }\end{array}$ & $\begin{array}{l}\text { Taofiq et al. } \\
\text { (2016), Gashaw et } \\
\text { al. (2020), Melinda } \\
\text { et al. (2020) }\end{array}$ \\
\hline Pleurotus eous & $\begin{array}{l}\text { Bacillus subtilis }\left(8.1^{\mathrm{b}}\right), \text { Escherichia coli }\left(11.1^{\mathrm{b}}\right) \text {, } \\
\text { Klebsiella pneumonia }\left(10.2^{\mathrm{b}}\right) \text {, Staphylococcus aureus } \\
\left(12.3^{\mathrm{b}}\right)\end{array}$ & Acidic polysaccharide & $\begin{array}{l}\text { Gunasekaran et al. } \\
(2021)\end{array}$ \\
\hline $\begin{array}{l}\text { Psathyrella } \\
\text { candolleana }\end{array}$ & Staphylococcus aureus $\left(0.016^{c}\right)$ & Psathyrelloic acid & Liu et al. (2019) \\
\hline $\begin{array}{l}\text { Ramaria } \\
\text { ephemeroderma }\end{array}$ & Sphaeropsis sapinea $\left(63.89 \%{ }^{\mathrm{a}}\right)$ & $\begin{array}{l}\text { Fermentation filtrate } \\
\text { extract }\end{array}$ & Qi \& Song (2006) \\
\hline $\begin{array}{l}\text { Rhizopogon } \\
\text { luteolus }\end{array}$ & $\begin{array}{l}\text { Agrobacterium tumefaciens }\left(6^{\mathrm{b}}\right), \text { Bacillus licheniformis } \\
\left(6^{\mathrm{b}}\right), \text { Bacillus subtilis }\left(13^{\mathrm{b}}\right)\end{array}$ & Chloroform extract & Sadi et al. (2015) \\
\hline Russula sp. & $\begin{array}{l}\text { Aspergillus niger, Aspergillus oryzae, Bacillus subtilis, } \\
\text { Escherichia coli, Saccharomyces Carlsbrgensis, } \\
\text { Staphylococcus aureus }\end{array}$ & $\begin{array}{l}\text { Ethanol extract, water } \\
\text { extract, }\end{array}$ & Chen \\
\hline Termitomyces sp. & Pseudomonas aeruginosa & Water extract & $\begin{array}{l}\text { Mariselvi \& } \\
\text { Ninganna (2018) }\end{array}$ \\
\hline $\begin{array}{l}\text { Trametes spp. } \\
\text { (Kakamega forest) }\end{array}$ & $\begin{array}{l}\text { Candida albicans }\left(0.83^{c}\right) \text {, Candida parapsilosis }\left(0.67^{c}\right) \text {, } \\
\text { Escherichia coli }\left(0.83^{c}\right) \text {, Klebsiella pneumoniae }\left(0.67^{c}\right) \text {, } \\
\text { methicillin resistant Staphylococcus aureus }\left(0.83^{c}\right) \text {, } \\
\text { Pseudomonas aeruginosa }\left(1.00^{c}\right), \text { Staphylococcus aureus } \\
\left(0.50^{c}\right)\end{array}$ & Hot & $\begin{array}{l}\text { Gebreselema et al. } \\
\text { (2019) }\end{array}$ \\
\hline $\begin{array}{l}\text { Trametes spp. } \\
\text { (Arabuko-Sokoke } \\
\text { forest) }\end{array}$ & $\begin{array}{l}\text { Candida albicans }\left(0.83^{c}\right) \text {, Candida parapsilosis }\left(0.83^{\mathrm{c}}\right) \text {, } \\
\text { Escherichia coli }\left(0.83^{\mathrm{c}}\right) \text {, Klebsiella pneumoniae }\left(0.83^{\mathrm{c}}\right) \text {, } \\
\text { methicillin resistant Staphylococcus aureus }\left(0.83^{\mathrm{c}}\right) \text {, } \\
\text { Pseudomonas aeruginosa }\left(0.83^{\mathrm{c}}\right), \text { Staphylococcus aureus } \\
\left(0.67^{\mathrm{c}}\right)\end{array}$ & Hot water extracts & $\begin{array}{l}\text { Gebreselema et al. } \\
(2019)\end{array}$ \\
\hline Tricholoma sp. & Staphylococcus aureus $\left(31^{\mathrm{b}}\right)$ & Polysaccharide & Hu \& Liu (2006) \\
\hline $\begin{array}{l}\text { Tricholoma } \\
\text { fracticum }\end{array}$ & $\begin{array}{l}\text { Agrobacterium tumefaciens }\left(6^{\mathrm{b}}\right) \text {, Bacillus licheniformis } \\
\left(6^{\mathrm{b}}\right) \text {, Bacillus subtilis }\left(6^{\mathrm{b}}\right) \text {, Staphylococcus aureus }\left(9^{\mathrm{b}}\right)\end{array}$ & Acetone extract & Sadi et al. (2015) \\
\hline $\begin{array}{l}\text { Xylobolus } \\
\text { princeps }\end{array}$ & Staphylococcus aureus $\left(0.4^{\mathrm{c}}\right)$ & Ethanol extract & $\begin{array}{l}\text { Tamrakar et al. } \\
(2017)\end{array}$ \\
\hline
\end{tabular}

$\mathrm{a}$, inhibition rate; $\mathrm{b}$, inhibition zone $(\mathrm{mm})$; c, minimal inhibitory concentration $(\mathrm{mg} / \mathrm{mL})$.

$\mathrm{IC}_{50}$ is not listed here because it is not the primary indicator of antimicrobial efficacy.

In summary, polysaccharides are the main active compound produced by mushrooms that are capable of inhibiting the proliferation of bacteria and viruses. Additionally, antibiotics, fatty acids, gallic acid, methionine, proteins, sulfides and toxins produced by mushrooms can exert antibacterial and antiviral effects (Tab 1). However, most studies to date have focused on the antibacterial and antiviral effects of mixed extracts, rather than specific components. Therefore, such active ingredients warrant further investigation.

\section{Antibacterial and antiviral mechanism of mushrooms}

Antibiotics can kill bacteria via several different mechanisms. For example, they can inhibit 
bacterial cell wall synthesis, resulting in the rupture and death of bacterial cells (Yan \& Bassler 2019). Such antibiotics include penicillin and cephalosporins (Mishra \& Kasik 1970). The growth and reproduction of bacteria also require specific osmotic pressure and $\mathrm{pH}$. Some antibiotics can alter cell membrane of permeability, creating an imbalance in the osmotic pressure across the cell. Both polymyxin and Brevibacterium act in this way ( $\mathrm{Yu}$ et al. 2015). Other antibiotics interfere with synthesis, including aminoglycosides and fosfomycin (Cai et al. 2009).

Viruses parasitize the host and depend on host cells for replication and proliferation. Antiviral drugs generally interfere with specific aspects of the viral replication cycle, such as virus adsorption, entry into cells, biosynthesis and release, and enhance host antiviral capabilities (Graci \& Cameron 2006). Amantadine and maraviroc (Wang \& Lu 2008) are penetration and shelling inhibitors, while saquinavir is a protein inhibitor.

Lentinus edodes (Berk.) Sing polysaccharide (Zhang et al. 2006, Zou et al. 2007) is known to be an enhancer and regulator of biological reaction enhancer and regulator. It can not only enhance humoral and cellular immunity nonspecifically but also exert immunomodulatory effects. Therefore, the antiviral mechanism of lentinan may lie in its ability to boost the immunity of infected cells, enhance the cell membrane stability, inhibit cytopathic effects and promote cellular repair (Zhang et al. 2007). AbM is rich in $\beta$-glucan, sterols and other components that can regulate cellular and humoral immunity. It can activate immune cells and improve immune function (Wang et al. 2008). Experiments have confirmed that A. aegerita polysaccharide exerts similar antibacterial activities to amoxicillin, and they both exhibit similar inhibitory effects on bacteria, but not mold. Penicillin, the main component of amoxicillin, is a $\beta$-lactam antibiotic that inhibits the synthesis of the bacterial cell wall by binding to bacterial cell membrane proteins that participate in the synthesis of peptidoglycan (Xin et al. 2011). It remains unclear whether the antibacterial mechanism of $A$. aegerita polysaccharide is similar to that of penicillin, and further research is thus warranted.

The antiviral mechanisms leveraged by mushrooms most likely relies on the ability of the active compounds to reduce or block viral adsorption and inhibit the replication of the virus in the host cell (Wang et al. 2008). Additionally, antiviral effects can be produced through improving the immunity of infected cells, enhancing the stability of cell membranes, inhibiting the development of cellular pathologies and promoting cell repair (Wang \& Wang 2011). The antibacterial mechanisms of mushrooms are mainly related to their ability to inhibit mycelial growth, spore germination and bacterial protein synthesis ( $\mathrm{Ji}$ et al. 2008). Polysaccharides produced by mushrooms can inhibit cell adhesion by forming a protective layer on the surface of cells or adhering to the surface of pathogens themselves. This ultimately prevents interactions between the pathogens and the surface of healthy cells to inhibit bacterial infection ( $\mathrm{Ji}$ et al. 2008, Hou et al. 2016, Dong et al. 2019). Furthermore, polysaccharides can inhibit bacterial growth by inhibiting bacterial defense enzymes and infection-related cell wall-degrading enzymes, or by destroying the bacterial cell membrane (Zhang et al. 2019). Additionally, the mushroom mycelium and the pathogenic bacteria can become enmeshed. Mycelial cell protoplasm containing pathogenic bacteria has been reported to become concentrated at the junction of two mycelial hyphae. Thus, the mycelium is likely acting in a beneficial manner to promote bacterial outflow. Additionally, shrinkage and breakage eventually disintegrate the pathogenic fungal hyphae to achieve an antibacterial effect (Zhang et al. 2014).

\section{Conclusion}

According to the studies discussed in this review, mushrooms can exert both bacteriostatic and antiviral effects. However, current research on the effects of mushrooms is limited. The wide repertoire of edible mushrooms has not yet been fully explored, and their active ingredients have not yet been fully characterized. Additionally, the mechanisms associated with the bacteriostatic and antiviral effects of mushrooms remain unclear. Therefore, further studies are warranted to clarify the effective components and the underlying mechanisms associated with the bacteriostatic and antiviral effects of mushrooms. 


\section{Acknowledgements}

This work was financed by the National Key Research \& Development Program of China (No. 2018YFE0107800).

\section{References}

Angelini P, Girometta C, Tirillini B, Moretti S et al. 2019 - A comparative study of the antimicrobial and antioxidant activities of Inonotus hispidus fruit and their mycelia extracts. International Journal of Food Properties 22 (1), 768-783.

Cai Y, Fan Y, Wang R, An MM et al. 2009 - Synergistic effects of aminoglycosides and fosfomycin on Pseudomonas aeruginosa in vitro and biofilm infections in a rat model. Journal of Antimicrobial Chemotherapy 64 (3), 563-566.

Chen QY, Miao CG, He HQ. 2012 - Study on extraction conditions and antibacterial activity of polysaccharide of artificial cultivation of Hericium erinaceus. Journal of Biology 29 (04), 8991.

Chen XJ, Gan YK, Luo YM. 2008 - Study on the bacteriostatic action of extract from the fruitbodies of Russula. Journal of Anhui Agri (10), 4138-4139.

Chowdhury MMH, Kubra K, Ahmed SR. 2015 - Screening of antimicrobial, antioxidant properties and bioactive compounds of some edible mushrooms cultivated in Bangladesh. Annals of Clinical Microbiology and Antimicrobials 14 (1), 1-8.

Dai XD, Zhang JC, Kong XH, Li YJ. 2007 - Preliminary research on antimicrobial effect of Agaricusblazei Murr. Edible Fungi of China (06), 36-37.

Dasgupta A, Acharya K. 2019 - Mushrooms: an emerging resource for therapeutic terpenoids. 3 Biotech 9(10), 369.

De Silva DD, Rapior S, Hyde KD, Bahkali AH. 2012 - Medicinal mushrooms in prevention and control of diabetes mellitus. Fungal Diversity 56 (1), 1-29.

Dong L, Han M, Liang HW, Zheng YX. 2019 - Comparative study on the bacteriostatic effect of fermentation broth and bran extract of common edible fungi. Journal of Guangzhou City Polytechnic 13 (02), 26-30.

Elena VM, Talía H-P, Octavio P-L. 2015 - Edible mushrooms: improving human health and promoting quality life. International journal of microbiology 2015, 376387.

Fogarasi M, Diaconeasa ZM, Pop CR et al. 2020 - Elemental composition, antioxidant and antibacterial properties of some wild edible mushrooms from Romania. Agronomy-Basel 10 (12), 1-9.

Friedman M. 2016 - Mushroom polysaccharides: chemistry and antiobesity, antidiabetes, anticancer, and antibiotic properties in cells, rodents, and humans. Foods 5 (4), 80.

Gashaw G, Fassil A, Redi F. 2020 - Evaluation of the antibacterial activity of Pleurotus spp. cultivated on different agricultural Wastes in Chiro, Ethiopia. International Journal of Microbiology 2020, 9312489.

Gebreselema G, Andrew N, Christine B, Desta B S. 2019 - Determination of antimicrobial activity of extracts of indigenous wild mushrooms against pathogenic organisms. Evidence-based complementary and alternative medicine 2019, 6212673.

Graci JD, Cameron CE. 2006 - Mechanisms of action of ribavirin against distinct viruses. Reviews in Medical Virology 16 (1), 37-48.

Gui FJ. 2004 - Antiviral activity of N-benzoylphenylisoserine sesquiterpenes from mushrooms of the genus Lactitus in vitro. World Ptomedicines (03), 115.

Gunasekaran S, Govindan S, Ramani P. 2021 - Sulfated modification, characterization and bioactivities of an acidic polysaccharide fraction from an edible mushroom Pleurotus eous (Berk.) Sacc. Heliyon 7 (1), e05964.

Hatvani N. 2001 - Antibacterial effect of the culture fluid of Lentinus edodes mycelium grown in submerged liquid culture. International Journal of Antimicrobial Agents 17 (1), 71-74. 
Hetland G, Johnson E, Bernardshaw SV et al. 2021 - Can medicinal mushrooms have prophylactic or therapeutic effect against COVID-19 and its pneumonic superinfection and complicating inflammation? Scandinavian Journal of Immunology 93 (1), e12937.

Hirasawa M, Shouji N, Neta T, Fukushima K et al. 1999 - Three kinds of antibacterial substances from Lentinus edodes (Berk.) Sing. (Shiitake, an edible mushroom). International Journal of Antimicrobial Agents 11 (2), 151-157.

Hou AP, Zhang SM. 2015 - Investigation on the universality of antibiotic property of polysaccharide in lentinan. Journal of Pharmaceutical Research 34 (04), 199-201.

Hou LD, Wu Y, Zhang J. 2016 - Inhibition effect of fermented filtrates of Pleurotus eryngii on 3 plant fungi. Acta Agriculturae Zhejiangensis 28 (03), 469-473.

Hu SQ, Liu TG. 2006 - Extraction and test of active metabolites from Tricholoma matsutake. Journal of Anhui Agricultural University (04), 499-501.

Jerica S, Jože B, Jana E, Tanja D et al. 2020 - L-Amino acid oxidases from mushrooms show antibacterial activity against the phytopathogen Ralstonia solanacearum. Frontiers in microbiology $11,977$.

Ji HF, Zhang LW, Song RQ. 2008 - Inhibiting mechanism of the extraction of Lactarius vellereus fermenting liquid on Alternaria alternata in poplar. Journal of Beijing Forestry University (04), 146-149.

Ji RQ, Li Y, Song RQ. 2012 - Optimization of cultivation conditions and security analysis of antifungal substance from Amanita sp. Journal of Northeast Forestry University 40 (03), 98102.

Kombrink A, Tayyrov A, Essig A, Stockli M et al. 2019 - Induction of antibacterial proteins and peptides in the coprophilous mushroom Coprinopsis cinerea in response to bacteria. Isme Journal 13 (3), 588-602.

Li C, Zhang P, Xiao LG, Liang ZS et al. 2017 - Antimicrobial activities and antimicrobial stabilities of the extracts from Flammulina velutipes. Food Research and Development 38 (02), 29-33.

Lin CQ, Yan J, Wang YL, Chen JC et al. 2014 - Physical-chemical properties, isolation and identification of antimicrobial substances from Pleurotus tuber-regium fermenting borth. Natural Product Research and Development 26 (04), 534-538.

Liu FZ, Wei J, Niu XM. 2003 - Study on antibacterial action of Coprircus comatus and the preservation of cherry. Food Science and Technology (05), 89-91.

Liu Y. 2009 - In vitro antibacterial activity of three edible fungi crude polysaccharides. Edible fungi 31 (02), 66-68.

Liu YP, Dai Q, Pu CJ, Wang M et al. 2019 - Psathyrellanic acid, a monocyclic diterpenoid from the basidiomycete Psathyrella candolleana. Natural Product Communications 14(6), 1-4.

Mariselvi M, Ninganna E. 2018 - Molecular identification and screening of mushrooms for antibacterial property against Pseudomonas aeruginosa and Staphylococcus aureus. Journal of Applied and Natural Science 10 (2), 791-796.

Martel J, Ojcius DM, Chang CJ, Lin CS et al. 2017 - Anti-obesogenic and antidiabetic effects of plants and mushrooms. Nature Reviews Endocrinology 13 (3), 149-160.

Melinda F, Maria DZ, Rodica PC, Szabolcs F et al. 2020 - Elemental composition, antioxidant and antibacterial properties of some wild edible mushrooms from Romania. Agronomy 10 (12), 1972.

Mishra RK, Kasik JE. 1970 - The mechanism of mycobacterial resistance to penicillins and cephalosporins. Internationale Zeitschrift fur klinische Pharmakologie, Therapie, und Toxikologie International journal of clinical pharmacology, therapy, and toxicology 3 (1), $73-77$.

Muszynska B, Grzywacz-Kisielewska A, Kala K, Gdula-Argasinska J. 2018 - Anti-inflammatory properties of edible mushrooms: A review. Food Chemistry 243, 373-381.

Nowacka N, Nowak R, Drozd M, Olech M et al. 2017 - Antibacterial, antiradical potential and phenolic compounds of thirty-one polish mushrooms. PLoS ONE 10 (10), e0140355. 
Pala SA, Wani AH, Ganai BA. 2019 - Antimicrobial potential of some wild Macromycetes collected from Kashmir Himalayas. Plant Science Today 6 (2), 137-146.

Qi JY, Song RQ. 2006 - Inhibiting effect of toxic mushroom strains and their toxins on pathogen of shoot blight of Pinus sylvestris var. mongolica (Sphaeropsis sapinea). Forestry Science \& Technology (03), 20-23.

Reis FS, Barros L, Martins A, Ferreira ICFR. 2012 - Chemical composition and nutritional value of the most widely appreciated cultivated mushrooms: An inter-species comparative study. Food and Chemical Toxicology 50 (2), 191-197.

Reis FS, Martins A, Helena Vasconcelos M, Moreles P et al. 2017 - Functional foods based on extracts or compounds derived from mushrooms. Trends in Food Science \& Technology 66, $48-62$.

Sadi G, Emsen B, Kaya A, Kocabas A et al. 2015 - Cytotoxicity of some edible mushrooms extracts over liver hepatocellular carcinoma cells in conjunction with their antioxidant and antibacterial properties. Pharmacognosy Magazine 11 (42): S6-S18.

Sevindik M. 2020 - Antioxidant and antimicrobial capacity of Lactifluus rugatus and its antiproliferative activity on A549 cells. Indian Journal of Traditional Knowledge 19 (2), $423-$ 427.

Song RQ, Ji RQ. 2005 - Inhibiting effects of 4 toxic mushroom strains and their toxin on Cytospora chrysosperma (Pers.) Fr. Journal of Beijing Forestry University (02), 88-91.

Suwannarach N, Kumla J, Sujarit K, Pattananandecha T et al. 2020 - Natural bioactive compounds from fungi as potential candidates for protease inhibitors and immunomodulators to apply for coronaviruses. Molecules 25 (8), 1800-1821.

Tamrakar S, Nishida M, Amen Y, Tran HB et al. 2017 - Antibacterial activity of Nepalese wild mushrooms against Staphylococcus aureus and Propionibacterium acnes. Journal of Wood Science 63 (4), 379-387.

Tang Q, Yang JD, Fang Ling, Hu W. 2018 - Study on antioxidative and antimicrobial activities of Lentinan. Journal of Anyang Institute of Technology 17 (02), 102-106.

Tang P, Cheng X, Yang Y. 2015 - Inhibitory activity of toxin extract from five kinds of Amanita against several plant pathogenic fungi. Journal of Yunnan Agrical Tural University (Natural Science) 30 (05), 681-685.

Taofiq O, Heleno SA, Calhelha RC, Alves MJ et al. 2016 - Development of mushroom-based cosmeceutical formulations with anti-inflammatory, anti-tyrosinase, antioxidant, and antibacterial properties. Molecules 21(10), 1372.

Tehrani MHH, Fakhrehoseini E, Nejad MK, Mehregan H et al. 2012 - Search for proteins in the liquid extract of edible mushroom, Agaricus bisporus, and studying their antibacterial effects. Iranian Journal of Pharmaceutical Research 11 (1), 145-150.

Wang J, Wang KY. 2011 - Discussion on the mechanism of resistance of the polysaccharide of Lentinus edodes against pathogenic bacteria and virus of plant. Agriculture 50 (01), 16-19.

Wang X, Wang XL, Qiu LY. 2008 - Research tendency in antiviral activity of Agaricus blazei Murill. Strait Pharmaceutical Journal 20 (12), 1-3.

Wang XM, Zhang J, Wu LH, Zhao YL et al. 2014 - A mini-review of chemical composition and nutritional value of edible wild-grown mushroom from China. Food Chemistry 151, 279-285.

Wang Y, Liao ZM, Yao Z, Lin PC. 2019 - Isolation and content determination of antimicrobial constituent in Agaricus gennadii. Journal of Natural Science of Hunan Normal University 42 (03), 70-74.

Wang YL, Bao HY, Xu L, Bau T. 2011 - Determination of main peptide toxins from Amanita pallidorosea with HPLC and their antifungal action on Blastomyces albicans. Acta Microbiologica Sinica 51 (09), 1205-1211.

Wang ZY, Lu HZ. 2008 - The new HIV drug- the CCR5 antagonist, Maravero. Chin J AIDS STD (04), 431-434.

Wasser SP. 2010 - Medicinal mushroom Science: History, Current status, Future trends, and unsolved problems. International Journal of Medicinal Mushrooms 12 (1), 1-16. 
Wasser SP. 2017 - Medicinal mushrooms in human clinical studies. Part I. Anticancer, Oncoimmunological, and Immunomodulatory activities: A Review. International Journal of Medicinal Mushrooms 19 (4), 279-317.

Wei BY, Yu XY, Huang L, Teng JW. 2007 - Agaricus bisporus (Lange) Sing polysaccharide antibacterial reactive and to curb corruption in the food research. Food Science and Technology (04), 93-95.

Xin YJ, Fang SH, Wang XF, Mao HL. 2011 - The antibacterial effect of Agrocybe aegerita polysaccharide. Edible Fungi 33 (04), 64-65.

Yan J, Bassler BL. 2019 - Surviving as a Community: Antibiotic Tolerance and Persistence in Bacterial Biofilms. Cell Host \& Microbe 26 (1), 15-21.

Ye ZQ, Lin YX. 2001 - Preliminary study on the bacteriostatic effect of Agaricus brasiliensis. Food Science (04), 82-84.

Yu Z, Qin W, Lin J, Fang S et al. 2015 - Antibacterial mechanisms of polymyxin and bacterial resistance. Biomed Research International 2015, 679109.

Zhang FM, Zhang SQ, Sun F, Liu ZY et al. 2006 - Inhibitory effect of lentinan on influenza virus. Journal of Changchun University of Traditional Chinese Medicine (04), 11-12.

Zhang FM, Zhang SQ, Sun F, Liu ZY et al. 2007 - Inhibitory effect of lentinan on herpes simplex virus. Journal of Changchun University of Traditional Chinese Medicine (01), 17-18.

Zhang J, Zhang SW, Xu BL et al. 2014 - Determining antifungal spectrum and mechanism of Trichoderma longibrachiatum in vitro. Chinese Journal of Eco-Agriculture 22 (06), 661-667.

Zhang JJ, Li Y, Zhou T, Xu DP et al. 2016 - Bioactivities and health benefits of mushrooms mainly from China. Molecules, 21(7), 938.

Zhang XB, Lin LN, Zhang GC, Yang J et al. 2019 - Inhibitory effect and antimicrobial mechanism of Lysurus mokusin extracts on Botrytis cinerea. Journal of Jilin Agricultural University 41 (02), 161-167.

Zheng DD, Hu YY, Wang Q. 2016 - Recent research on bioactive components from Agaricus bisporus. Acta Edulis Fungi 23 (02), 94-103.

Zheng SY, Zhang QQ, Liu GQ, Lu YX. 2010 - Antibacterial activity of crude extracts from the fruiting body of a species of edible fungi. Jiangsu Agricultural Sciences (01), 340-341.

Zheng Y, Zou QQ, Zhang D, Wu JH et al. 2013 - Research progress on chemical composition and biological activities of Dictyophora indusiata. Journal of Food Science and Technology 31 (03), 39-45.

Zou HX, Zhang SQ, Liu ZY, Zhang FM et al. 2007 - Inhibitory effect of lentinan on Coxsackie group B virus. Chinese Journal of Control of Endemic Diseases (05), 351-352.

Zou L, Tan Y, Yang MB, Sun TT. 2012 - Antimicrobial activity of fermented product from Inonotus obliguus. Journal of Northeast Forestry University 40 (11), 123-126. 